\title{
Design and Analysis of LH Miniaturized Microstrip Filter Based on DNG
}

\author{
Shubhi Jain, Gloria Joseph \\ Department of Electronics and Communication Engineering, Swami Keshvanand Institute of Technology, \\ Management \& Gramothan, Jaipur, Rajasthan (INDIA) \\ Email: shubhijain19@gmail.com \\ Received 09.02.2021, Received in revised form 19.03.2021, Accepted 30.03.2021
}

\begin{abstract}
The antenna plays a key role in the wireless communication. The concept of dual mode band stop filter based on DNG using the Left Handed Meta materials is proposed which having the negative permeability and permittivity. These materials have a special unique property "negative refraction" and it is able to support the backward waves. In this paper $\mathrm{LH}$ Miniaturized Microstrip filter is presented. The $\mathbf{L H}$ filter has characteristic of narrow fractional bandwidth and reduced size in comparison to conventional one. The proposed filter physically implemented on top of a FR/4 'Glass/Epoxy' substrate which having the Dielectric constant (gr) is 4.4. The substrate thickness is $1.6 \mathrm{~mm}$ and having the Loss tangent of 0.02 using the conventional fabrication process. The frequency band is lie between 1.5 to 1.7 GHz. It gives the good results. There is reduction in the size of filter because there is no periodic structure is used that was used in SRR, CSRR and IDCLLR. The proposed design is five times smaller than the conventional one.
\end{abstract}

Keywords - Meta Materials, Filters, Resonator, IE3D, Patch Antenna

\section{INTRODUCTION}

Today the world is moving towards the wireless network. It is one of the fastest growing technologies in the world. It has increased the uses of microwave equipments. To coordinate with the development in wireless network, it is necessary to develop the new technology microwave equipments. The development of microwave equipment is dependent on the development of Microstrip filters, antennas and other devices. Modern devices are much fast than the last one. The innovation is applied to make device filters simpler and simpler, reconfigurable and lightweight [1]. The advanced new technology filters are in small size. So they are capable to reduce the area of device. Now the wireless devices are available in the market with small size and portable features working at different radio frequencies [2]. There are different parameters that contribute into the measurement of the dimension of the filters, i.e. Content substrates, thickness etc. of the material [3], [4], [5], [6]. These materials can minimize the dispersive loss effect at the high frequencies by developing the optical and microwave devices with negative permeability [7].
These materials are often referred to as materials of a wider class than LHM Metamaterials [8]. Such materials tend to manipulate dielectric permittivity [9]. DNM is essentially negative index Meta (NIM) materials, which has both are positive results in propagation in the forward direction. These materials able to allow the development of super lenses that can have a spatial resolution below the wavelength. It produces a negative backward wave if both permittivity and permeability are negative. In the resonance state, phase cancelation occurs. Resonance depends on thickness ratio; compactness is the main definition [10], [11],[12].

In this paper the proposed band reject filter was introduced on top of FR4 glass epoxy with a dielectric constant of $\varepsilon r=4.4$. This design efficiency reveals more than 80 percent of the filter miniaturization. The simulations are carried out on IE3D software and compared with the configuration of the hardware. Section II explains about the design parameters and physical specification parameters of the filter. Section III illustrates different simulation results. Section IV gives the conclusion of the work.

\section{DESIGN PARAMETERS}

The filter is fabricated using the appropriate material. The Band stop filter design is implemented by using the left-handed material. It is used for fabrication of filter because when a uniform wave is launched in this material, the direction of phase velocity is opposite to the pointing vector. So the concept of mixing slabs of vacuum dielectric materials and Metamaterials with both permeability and permittivity is based on phase transition. The filter system deals with the split ring resonators. The resonator size is approximately to the half wavelength of the high frequencies in free space. For simulation efficiency, different kinds of time domain methods are required. As in earlier SRR, CSRR for designing the filter, there is no periodic structure used in this miniaturized band reject filter. Compared to others, the size of proposed Miniaturized Micros trip Band Reject Filter Dual Mode is much smaller. In fig. 1(a) and fig. 1(b) dual mode miniaturized-based band-reject filter is shown. The proposed Dual-mode miniaturized band-reject micro strip filter has been designed to set the width 
of the central strip to $3.059 \mathrm{~mm}$ to make the impedance of the characteristics of the line approximately $50 \Omega$, so that both port sides experience relatively low loss levels of return.

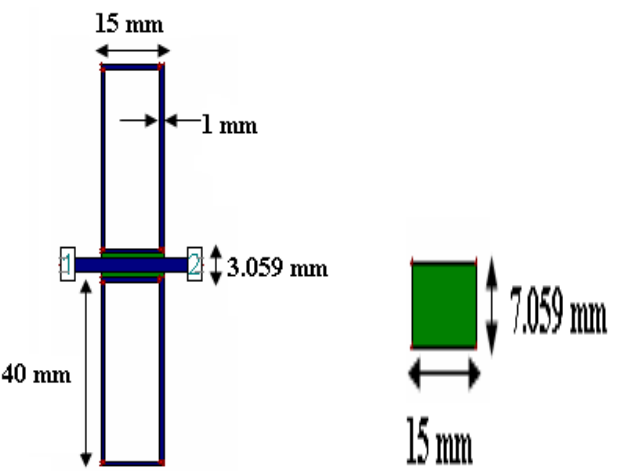

Fig. 1 (a). Dual Mode Miniaturized-based Band-reject Filters (left) (b). Dimensions of Rectangle cut on the Ground Layer (right)

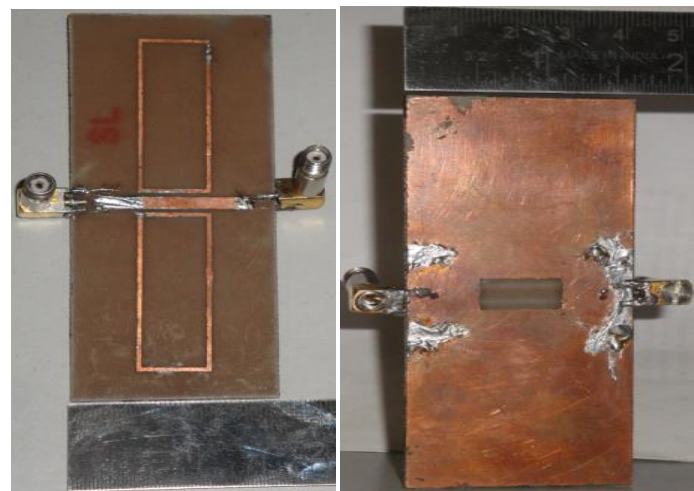

Fig. 2(a). Fabricated Dual Mode Band Reject Filter (Top Layer) (Left) (b). Fabricated Dual Mode Band Reject Filter (Ground Layer) (right)

Fig. 2(a) and fig. 2(b) shows fabrication of filter on top of a FR/4 'Glass/Epoxy' substrate with a dielectric constant (cr) of 4.4 with a thickness of 1.6 $\mathrm{mm}$ and a loss tangent of 0.02 by using the traditional manufacturing process. The antenna bandwidth is shown by materials only, not by the antenna size. This demonstrates a different form of antenna than the traditional one.

\section{SIMULATION RESULTS AND COMPARATIVE ANALYSIS}

In the proposed work a new significantly reduced-size L band LH micro strip dual mode notch band stop filter has been designed and fabricated. There is a center frequency of $1.65 \mathrm{GHz}$ in this filter. Although the rejection is approximately $-16 \mathrm{~dB}$ at the resonance frequency, the better matching of the LH segment is thought to increase it. With the help of a spectrum analyzer the scattering parameters are calculated. Using the Method of Moments (MoM) based electromagnetic solver IE3D commercial software; numerical measurements of the scattering parameters are done. Fig. 3(a) shows the Return Loss and fig. 3(b) shows Transmission Line Coefficient with respect to
Frequencies graph. These indicate calculated and simulated values of insertion and return losses. Single and double Metamaterials are distributed from this study. So that, these material parameters have negative values over the precise frequency band, and their values can be modified according to variation in frequency. The outcome is therefore focused on the dispersed design. The active materials have the potential to display the negative parameters over the precise frequency band.

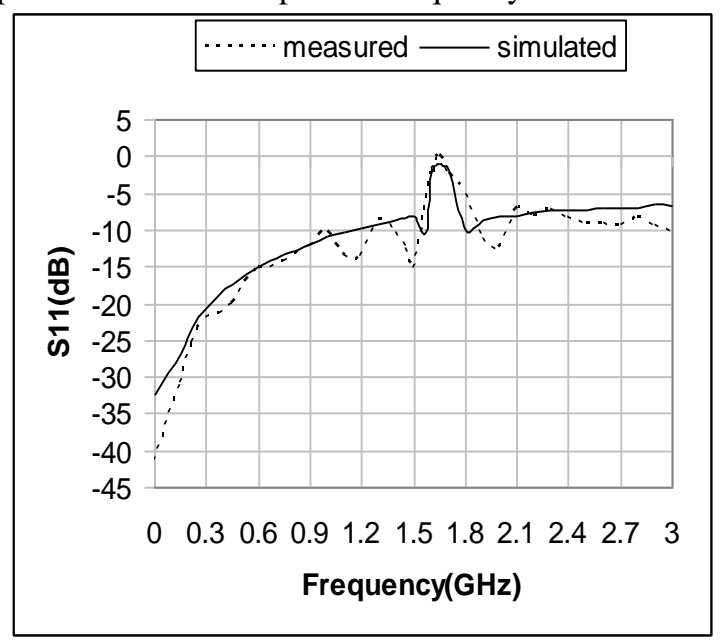

Fig. 3(a). Return loss Vs Frequency

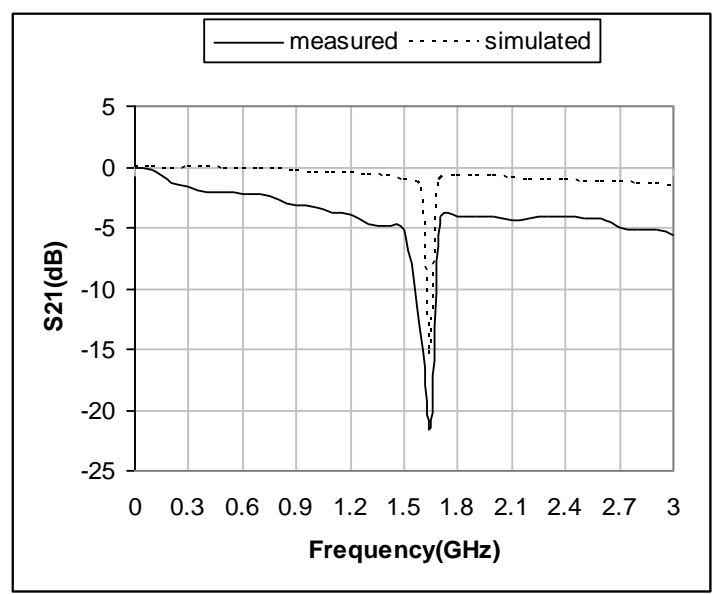

Fig. 3(b). Transmission Line Coffiecient
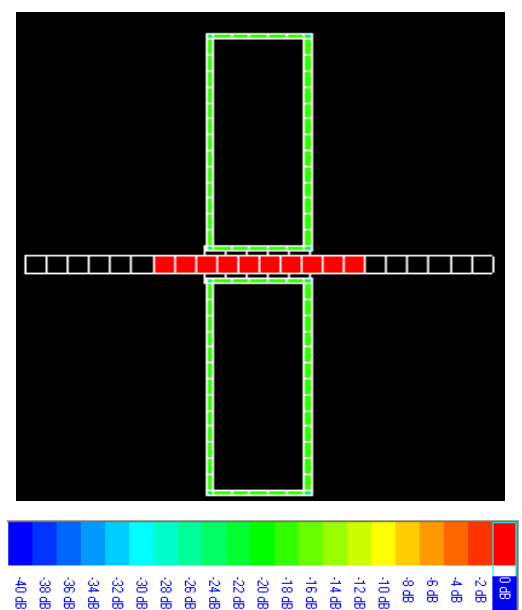

Fig. 4. Surface Current Distribution 
The substratum is divided into a wire-grid model which is a method for estimating the currents on the microwave transmission line. The near-field magnetic strength above the transmission line of the microwave is determined and measured. The magnetic field integral equation is defined on the basis of the magnetic near-field strength by applying the Green function. It is used to estimate the currents flowing on the microwave transmission line which is shown in Figure 4.

\section{CONCLUSION}

In this work, the band rejection filter is designed, fabricated and evaluated by using the IE3D software with high frequency. The center frequency of filter is $1.65 \mathrm{GHz}$, while at resonance frequency, rejection is above $-16 \mathrm{~dB}$. To achieve the $90^{\circ}$ phase shift, many traditional filters need $\lambda / 4$ resonator, which is a very large dimension, particularly in the RF range. Thus, the filter size of LH technology is significantly reduced. It should be able to reduce its size by more than $80 \%$. Due to the low cost and small size of the patch itself, new Metamaterials are demanding to get the output through optical devices in wireless communication. The Microstrip band rejection filter is analyzed and used on IE3D software with high frequency here. The filter center frequency is $1.65 \mathrm{GHz}$ while resonance frequency rejection is above $-16 \mathrm{~dB}$. To achieve a $90^{\circ}$ phase shift, many traditional filters require a $\lambda / 4$ resonator which is a very large dimension, particularly in the RF range. Composite left-handed Metamaterials are used in this Microstrip filter to achieve phase transition with the combination of dual negative Metamaterials. The double negative medium is partly loaded with it. Thus by using proposed filter size of the LH technology, it should be able to reduce the size. In future more reduction in the size can be performed.

\section{REFERENCES}

[1] Dwivedi Ved Vyas, Kosta Y P and Jyoti Rajiv, "Miniaturized Compact Microstrip Patch Antenna Designed on a Double Negative Metamaterials for RF wireless systems", Proc. International Conference on Recent Advances in Microwave Theory and Applications, Nov. (2019), Pages 83-88.

[2] R. S. T. Weiland, R. B. Greegor, C. G. Parazzoli and A. M initio "Numerical simulation of Left-Handed Metamaterials: Comparison of Calculations and Experiments", Journal of Appl. Physics, (2018),90

[3] Aieta, F., Kats M. A., Genevet P. and Capasso F., "Multi Wavelength Achromatic Meta Surfaces by Dispersive Phase Compensation", Science Magazine, (2015), Volume 3, Issue 6, Pages 1342-134.

[4] V. C. Bengin, V. Radonic and B. Jokanovic, Fractal Geometries of Complementary Split-Ring Resonators, IEEE Transaction on Microwave Theory and Techniques, Oct.( 2008), Volume 56, No. 10, pp. 2312-2321.

[5] Vendik, O. Vendic, I. Kolmakov and M . Odit, "Modeling of Isotropic Double Negative Media for Microwave Applications", Opto Electronics Review, (2006),Volume 14, No. 3, Pages 179-186.

[6] D. Ahn, J. S. Park, C. S. Kim, J. Kim, Y. Qian and T. Itoh, "A Design of the Low-pass Filter using the Novel Microstrip Defected Ground Structure," IEEE Trans. Microwave Theory Tech., Jan.(2001), Volume 49, Pages.86-93.

[7] X. Chen, T. M. Grzegorczyk, B. I. Wu, J. J. Pacheco and J. A. Kong, "Robust Method to Retrieve the Constitutive Effective Parameters of Metamaterials", Physical Review E 70 016608, The American Physical Society, (2004).

[8] R. Yang, Y. Xie, P. Wang and L. Li, "Microstrip Antennas with Left Handed Materials Substrates", Journal of Electromagnetic Waves and Application, (2006), Volume 20, No. 9, Pages 1221-1233.

[9] J. S. Lim, S. W. Lee, C. S. Kim, J. S. Park, D. Ahn and S. Nam, "A 4.1 Unequal Wilkinson Power Divider," IEEE Microwave and Wireless Components Letter, Mar. (2001) Volume 11, Issue 3, Pages 124-126.

[10] D. Ahn, J. S. Park, C. S. Kim, J. Kim, Y. Qian and T. Itoh, "A design of the Low-pass Filter using the Novel Microstrip Defected Ground Structure", IEEE Trans. Microwave Theory Tech., Jan.( 2001),Volume.49, Pages 86-93.

[11] C. Caloz and T. Itoh, "Transmission line approach of LeftHanded (LH) Materials and Microstrip Implementation of an Artificial LH Transmission line", IEEETrans.Antennas Propag.,May( 2004), Volume 52, No. 5, Pages 1159-1166.

[12] Garcia-Garcia, j., Bonache, j., Ignacio, G., Ferran, M., Velazquez-Ahumada, Maria del Castillo, and Martel, Jesus, "Miniaturized Microstrip and CPW Filter Using Coupled Metamaterial Resonators," IEEE Trans. Microw. Theory Tech., June (2018), Volume 54,No.6, Pages 2628-2635. 